\title{
Mutant mice know no fear
}

Is it possible that some people really don't know the meaning of the word fear? A new strain of risk-taking mice is helping scientists study the genetic foundations that underlie the brain's ability to recognize and remember risky or uncomfortable situations.

Researchers have long recognized the amygdala as a vital center for the processing of emotion, including responses such as fear perception, although there is as yet only incomplete understanding of these processes at the molecular level. Previous work from James Olson's lab at the Fred Hutchinson Cancer Research Center (Seattle, WA) has shown that neurogenic differentiation factor 2 (Neurod2), an essential transcription factor for neuronal development, seems to have an important function in the amygdala. Mice that entirely lack Neurod2 expression suffer from several neurological problems and die shortly after birth; on the other hand, mice with only one copy of this gene survive to adulthood but, as recently reported by Olson's group, show significant defects in amygdala development-with important behavioral ramifications (Proc. Natl. Acad. Sci. USA, 11 October).
Given the choice between 'protected' and 'unprotected' environments, wild-type mice spent $99 \%$ of their time in enclosed areas, whereas the heterozygotes spent almost 90\% of their time in the open, suggesting that these mice are far less risk-averse in unconditioned situations. The heterozygotes showed similar reductions in emotional learning in conditioned fear tests, where a negative stimulus (a mild shock) is preceded by an operant signal, suggesting reduced ability to build long-term memory relating to fear-inducing situations. The heterozygous mice showed developmental defects in the basolateral amygdala - a key center for emotional learning related to fear response-and Neurod2 seems to be a key switch for triggering the expression of genes essential to amygdala function. According to Olson, this is clear evidence of the importance of gene dosage for proper brain function. "It's not just whether you have it or not," he says. "You need the full amount to be a normal mouse."

Previous research has linked defects of the amygdala with problems in emotional processing, as well as disorders such as schizophrenia. These new findings on Neurod2's role in amygdala development and function

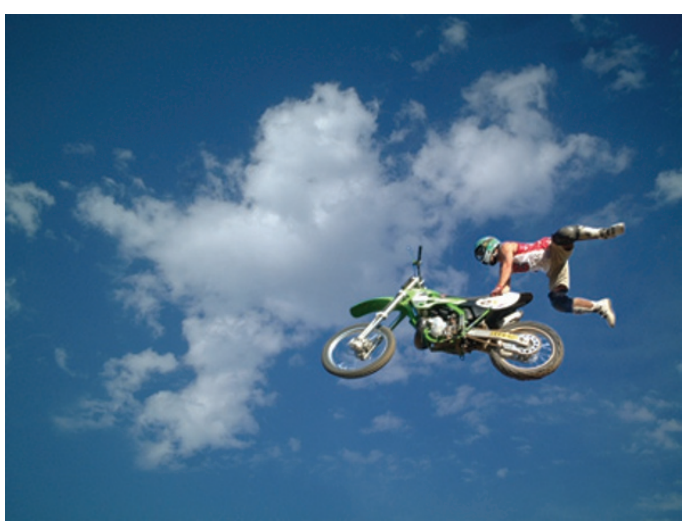

may provide exciting new opportunities for better understanding the effects of this structure on human behavior, and Olson says that his team is looking forward to extending their research to the analysis of Neurod2 gene variations: "If there are polymorphisms in the gene, then we could do a broader study to see if those polymorphisms match with people who have differences in emotional learning, or fear perception, or schizophrenia, or some of the other diseases that involve the amygdala."

\section{Michael Eisenstein}

\section{TO STOP CANCER USE YOUR BEAN?}

A compound found in beans, nuts, and wheat bran shows potent antitumor activity both in vitro and in vivo. This finding presents the possibility that a diet high in these foods may help prevent cancer; likewise, it may form the basis of new treatments for established tumors.

Angiogenesis - the formation of new blood vessels-involves the migration and proliferation of endothelial cells to form tubular structures able to carry blood. Angiogenesis is an important part of normal development and wound healing. It is also critical for the growth of tumors, creating an avenue for tumor cells to acquire oxygen and nutrients; indeed, without its own blood supply a tumor cannot grow much larger than the size of a pea.

Because of its involvement in a key angiogenesis pathway, the enzyme phosphoinositide 3-kinase (PI3K) is a desirable target for anticancer therapies. However, variables such as toxicity and insolubility have hindered the search for a safe and effective inhibitor.

Now, in the 15 September issue of Cancer Research, Marco Falasca of University College London's Sackler Institute (London, UK) and his colleagues report that inositol pentakisphosphate (Ins $\left.(1,3,4,5,6) P_{5}\right)$, a naturally occurring, water-soluble compound, specifically inhibited the activity of the enzyme. Falasca's team found that Ins $(1,3,4,5,6) \mathrm{P}_{5}$ inhibited PI3K activity in cultured endothelial cells, reduced endothelial cell migration in vitro, and blocked angiogenesis in mice. In mice with human ovarian cancer xenografts, the compound also reduced tumor growth as much as does cisplatin, a drug commonly used in treatment of ovarian cancers.

Unlike conventional chemotherapeutic agents, Ins $(1,3,4,5,6) \mathrm{P}_{5}$ did not seem to cause toxic side effects. As Falasca tells Lab Animal, "The possible use of nontoxic, natural compounds to target cancer cells is attractive. Furthermore, the observation that inositol polyphosphates are easily absorbed by oral administration makes [the compound] even more promising in terms of therapeutic potential."

In addition, because the chemical is found naturally in legumes and other foods, it seems reasonable that including these in your diet may help ward off the development of cancer.

Falasca's group thinks that Ins $(1,3,4,5,6) \mathrm{P}_{5}$ holds great promise as an anticancer treatment-either on its own or in combination with other drugs. They are currently gathering additional data in preparation for clinical trials.

Tanja Schub 